\title{
REVISTAMATERIA
}

ISSN 1517-7076 artigo 11485, pp.1144-1157, 2012

\section{Estudo do desempenho de filmes contendo sais de terras raras como inibidores de corrosão da liga ZnFe eletrodepositada sobre aço}

\author{
Study of the performance of films containing rare earth salts as \\ corrosion inhibitors of zinc-iron alloy electroplated steel
}

Dos Santos, M.C.G., Freire, C.M.A

Faculdade de Engenharia Mecânica, UNICAMP, C.P. 6122, cep 13083-970, Campinas, SP

e-mail: msantos22@zipmail.com.br ; celia@fem.unicamp.br

\section{RESUMO}

Aço revestido com eletrodepósitos de liga ZnFe é um material de uso comum na indústria de construção civil e automotiva. O revestimento à base de zinco necessita, entretanto, ser protegido de ambientes corrosivos através de um tratamento de superfície que geralmente contém cromo hexavalente $\left(\mathrm{Cr}^{+}\right)$, tóxico para o meio ambiente e cancerígeno para o homem. O presente trabalho apresenta um estudo com inibidores de corrosão contendo terras raras (Ce ou La), sobre aço revestido com eletrodepósitos de liga ZnFe. Esses filmes foram avaliados através das Curvas de Polarização, da Espectroscopia de Impedância Eletroquímica (EIE), da Microscopia Eletrônica de Varredura (MEV) e Espectrometria de Energia Dispersiva (EDS). A espectroscopia fotoelétrica de emissão de raios-X (XPS) também foi utilizada para comprovar a presença desses filmes contendo óxidos sobre a superfície da liga ZnFe. Os resultados de EIE e polarização indicaram que as camadas estudadas foram eficientes contra corrosão e que seu desempenho dependia do tipo de cátion presente no sal. A amostra representada por $\mathrm{Ce}+\mathrm{ZnFe}$ mostrou o melhor desempenho na proteção contra a corrosão, havendo um decréscimo de aproximadamente 10 vezes na densidade de corrente quando a liga foi medida com $\mathrm{Ce}+\mathrm{ZnFe}$ um aumento de IZI em uma ordem de grandeza.

Palavras-chave: corrosão, liga $\mathrm{ZnFe}$, terras raras, XPS

\section{ABSTRACT}

Zinc-iron alloy electroplated steel is a material commonly used in the construction industries and automotive. This zinc-based material needs, however, to be protected against corrosive environments through a surface treatment that generally has hexavalent chromium, wich is toxic to the environment and may cause cancer to human being. This paper shows a study with the films of rare earth ( $\mathrm{Ce}$ or $\mathrm{La}$ ) on the zinc-iron alloy electroplated steel. These films were evaluated using Polarization Curves, Electrochemical Impedance Spectroscopy (EIS), Scanning Electron Microscopy (SEM) and EDS. The X-ray photoelectron spectroscopy (XPS) was also used to prove the existence of oxide films on the surface of zinc-iron alloy electroplated steel. The EIS and Polarization results have shown that the systems under study were efficient against corrosion and its performance depended upon the type of salt. The sample $\mathrm{ZnFe}+\mathrm{Ce}$ proved to have the best inhibitors against corrosion. The cathodic branch of the polarization curve presents a decrease in order of 10 times in the current density when the ZnFe alloy coating were treated with rare earth and IZI increase in order of one time at the same circumstance.

Keywords: corrosion, zin-iron alloy, rare earth, XPS. 


\section{INTRODUÇÃO}

A utilização do zinco ou ligas de zinco como forma de proteção catódica em aços é uma prática consolidada atualmente, pois os aços revestidos com zinco possuem uma ampla faixa de aplicação na indústria da construção civil e na produção de peças na indústria automotiva. Esses materiais, no entanto, não apresentam boa proteção em ambientes corrosivos como intemperismo urbano, necessitando de tratamento com inibidores tais como, cromatos, fosfatos e íons de terras raras (TR) que retardam esse processo de degradação do material.

Inibidores de corrosão são geralmente aplicados sobre metais e ligas objetivando proteger a superfície contra a corrosão. Esses inibidores produzem uma fina camada sobre o metal a ser protegido, conhecida como camada passivadora. Portanto, há crescente interesse no desenvolvimento de processo de passivação alternativos a cromatização para que se possa cessar o uso de $\mathrm{Cr}^{+6}$ [1-5]. Esses procedimentos alternativos, tais como camadas de passivação por terras raras , molibidados e revestimentos obtidos pelo processo sol-gel apresentam seus prós-contras quando se leva em consideração propriedades de resistência a corrosão, aderência a revestimentos orgânicos, resistência a fadiga e uniformidade do depósito. [6]

Os inibidores passivantes, como os cromatos, por exemplo, agem como barreira, uma vez que suprimem as reações catódicas, formando óxidos insolúveis nas regiões de corrosão ativa ou em toda a superfície, limitando a permeabilidade do eletrólito e reduzindo a taxa de corrosão de acordo com a reação [7, $\underline{8}$, 9]:

$$
3 \mathrm{Zn}+2 \mathrm{CrO}_{4}=+2 \mathrm{H}^{+} \quad 3 \mathrm{ZnO}+\mathrm{Cr}_{2} \mathrm{O}_{3}+2 \mathrm{OH}^{-}
$$

Apesar de ser um excelente protetor de corrosão o uso de cromatos e outros compostos contendo Cr6+ tem sido limitado desde 1982 e a diretiva de 2000/53/EC do Parlamento Europeu proibiu o uso de cromo hexavalente desde 2007 [1]. Entre as alternativas possíveis ao uso do Cr6+, os tratamentos com o uso de sais de terras raras têm sido uma solução viável. Os sais de terras raras foram usados com sucesso em muitos metais e ligas para proteção contra a corrosão em ambientes aquosos. Referências com o uso de cloretos e nitratos de cério e lantânio para inibição de corrosão em ligas de alumínio [2, 3] no zinco e ligas de zinco e ferro $[\underline{6}, \underline{8}, \underline{9}, \underline{10}, \underline{11}, \underline{12}$,] ligas de $\mathrm{NdFeB}[5]$, são encontradas na literatura relatando bons resultados. A passivação com as terras raras pode ser feita por imersão [10,11] por eletrodeposição [13].

MONTEMOR et al. [10] e TAMBORIM et al [14], relatam que os tratamentos baseados em $\mathrm{La}(\mathrm{NO} 3) 3$ são mais eficazes do que os tratados com $\mathrm{Ce}(\mathrm{NO} 3) 3 \mathrm{ou}$ em $\mathrm{Y}(\mathrm{NO} 3) 3$. O mecanismo de inibição sobre zinco com tratamento de sais de cério também foi investigado por ARAMAKI [7], que em seus estudos revela a formação de uma camada rica em hidróxido de cério, formado pela adsorção na superfície hidroxilada do zinco. Esse processo conduz à formação de $\mathrm{Ce} 2 \mathrm{O} 3$ na superfície do zinco que inibe as reações catódicas.

Nesse contexto pode-se dizer que o mecanismo de atuação das terras raras parece estar baseado no bloqueio das áreas catódicas, reduzindo a taxa de processos catódicos e como consequência, também os processos anódicos $[7, \underline{9}, \underline{11}, \underline{12}, \underline{14]}$, conduzindo ao aumento de proteção contra a corrosão além de serem ambientalmente corretos.

Levando em consideração o crescente consumo de eletrodepósitos de ligas de zinco em construções civis, na indústria aérea e automotiva esse artigo procura fornecer informações sobre o comportamento de filmes contendo óxidos de terras raras como inibidores em processos corrosivos na interface entre os filmes e a liga $\mathrm{ZnFe}$ através da caracterização dessas amostras por EIE e polarização. 


\section{MATERIAIS E MÉTODOS}

O procedimento experimental foi dividido em quatro etapas: a primeira etapa consistiu da preparação da superfície metálica (eletrodeposição de camada de liga $\mathrm{ZnFe}$ ), a segunda da preparação e aplicação das soluções contendo terras raras sobre a superfície da liga $\mathrm{ZnFe}$, e a terceira etapa é constituída pela caracterização elementar feita por XPS seguida pela caracterização eletroquímica dos sistemas.

O substrato metálico utilizado para deposição dos filmes contendo as terras raras é o aço carbono com área de 19,6 revestido com liga $\mathrm{ZnFe}$. A camada de liga $\mathrm{ZnFe}$ foi obtida através do processo de eletrodeposição por corrente pulsada, utilizando banhos comerciais fornecidos pela empresa Surtec.

\subsection{Preparação da Superfície Metálica}

A superfície da liga $\mathrm{ZnFe}$ foi limpa em solução contendo detergente neutro comercial por 2 minutos, depois lavagem dupla com água de torneira por 0,5 minuto, seguido por lavagem dupla com água deionizada por 0,5 minuto. Depois disso a superfície da liga $\mathrm{ZnFe}$ foi mergulhada nas soluções contendo nitrato de cério ou lantânio.

O preparo das soluções contendo terras raras e sua aplicação estão descritos a seguir.

\subsubsection{Soluções de Terras Raras:}

As soluções $\mathrm{Ce}(\mathrm{NO} 3)_{3}$ e de $\mathrm{La}(\mathrm{NO} 3)_{3}$, foram preparadas na concentração de $0,01 \mathrm{M}$ a temperatura ambiente.

\subsubsection{Aplicação das Soluções de Terras Raras}

A Tabela 1 apresenta as amostras em estudo e os tratamentos realizados de acordo com a etapa abaixo exposta:

1. Somente imersão na solução de nitrato de cério durante 4,5 minutos e secagem na estufa a $100^{\circ} \mathrm{C} \pm 5$ por 40 minutos e na sequência secagem à temperatura ambiente por 24 horas.

2. Somente imersão na solução de nitrato de lantânio durante 4,5 minutos e secagem na estufa a $100^{\circ} \mathrm{C} \pm 5$ por 40 minutos e na sequência secagem à temperatura ambiente por 24 horas.

A temperatura de $100^{\circ} \mathrm{C}$ por 40 min foi adotada para não alterar a morfologia da liga $\mathrm{ZnFe}$, que sofre alterações importantes em suas propriedades contra corrosão quando submetido a temperaturas superiores à $110^{\circ} \mathrm{C}[15]$.

Tabela 1: Amostras e respectivos tratamentos sofridos.

\begin{tabular}{l|c|c}
\hline \multirow{2}{*}{ AMOSTRAS } & \multicolumn{2}{|c}{ TRATAMENTOS } \\
\cline { 2 - 3 } & 1 & 2 \\
\hline Znfe & --- & --- \\
\hline ZnFe+Ce & $X$ & --- \\
\hline ZnFe+La & --- & $X$ \\
\hline
\end{tabular}

\subsection{Análise de XPS (Espectroscopia Fotoelétrica de Emissão de Raios-X)}

Os espectros de XPS para as amostras analisadas foram obtidos com um espectrometro VSW HA100, operando com analisador hemisférico em modo de transmissão constante, o que resulta numa largura da linha para $\mathrm{Au} 4 \mathrm{f} 7 / 2$ de 1,6eV. Para excitação dos elétrons foi utilizada radiação $\mathrm{K} \alpha$ do $\mathrm{Al}, 1486,6 \mathrm{eV}$. A pressão durante as medidas foi sempre inferior a $2 \times 10-8 \mathrm{mBar}$. Efeitos de carregamento foram corrigidos através da linha de C1s cuja energia de ligação foi fixada em $284,6 \mathrm{eV}$. A perpendicular à superfície da amostra foi paralela ao eixo do analisador para todas as análises. 


\subsection{Ensaio de Espectroscopia de Impedância Eletroquímica (EIE) e Curvas de Polarização}

O comportamento eletroquímico dos filmes de terras raras foram estudados por EIE sobre o potencial de circuito aberto na faixa de frequência de $10-2$ a $105 \mathrm{~Hz}$ e 5 pontos por década, utilizando-se um potenciostato modelo 273A da EG\&G em conjunto com um analisador de frequência Lock-in PAR 5210. Para o gerenciamento dos ensaios e aquisição dos dados foi utilizado o software PowerSine (PAR) . A amplitude da função senoidal de tensão usada foi de $10 \mathrm{mV}$. A célula de três eletrodos para amostras planas, continha um eletrodo de referência de calomelano saturado (SCE), uma grande grade de platina como contra-eletrodo e eletrodo de trabalho formado pela liga de $\mathrm{ZnFe}$ com área de 19,6 cm2 revestida ou não pelos filmes contendo TR (terras raras). Uma solução $0,05 \mathrm{M}$ de $\mathrm{NaCl}$ foi usada como eletrólito para caracterizar as camadas.

As curvas de polarização das amostras e da liga $\mathrm{ZnFe}$ foram obtidas em solução de $\mathrm{NaCl}$ 0,05M usando o eletrodo de calomelano saturado como referência e platina como contra eletrodo. As curvas de polarização foram inicialmente obtidas num intervalo de $-250 \mathrm{mV}$ a $250 \mathrm{mV}$ em relação ao potencial de circuito aberto numa velocidade de $0,166 \mathrm{mV} / \mathrm{s}$. Para separar as curvas de polarização potenciodinâmica anódica e catódica novas curvas foram levantadas num intervalo de - $-1,0$ a $0,0 \mathrm{~V}$ e $-1,0$ a $-2,0 \mathrm{~V}$ em relação ao ESCE na velocidade $0,166 \mathrm{mV} / \mathrm{s}$, respectivamente. Os ensaios foram realizados à temperatura ambiente e meio naturalmente aerado.

\subsection{Microscopia Eletrônica de Varredura}

A superfície das amostras foi observada com um microscópio eletrônico de varredura JEOL JXA-8040A com uma tensão de aceleração de $5 \mathrm{keV}$ e $25 \mathrm{keV}$ e uma emissão de filamento de $200 \mu \mathrm{A}$. Para confirmação da presença dos depósitos de terras raras foi realizado concomitantemente analises de EDS, determinando de forma semiquantitativa a porcentagem atômica dos elementos químicos presentes em cada fase do sistema.

\section{RESULTADOS}

Neste ítem serão apresentados os resultados obtidos na caracterização dos filmes de terras raras aplicados como inibidores de corrosão na superfície do $\mathrm{ZnFe}$ eletrodepositada sobre aço, bem como a discussão dos mesmos de maneira a se conhecer qual dos filmes apresentaram as melhores características de proteção contra corrosão.

Serão apresentados os resultados de XPS para a liga $\mathrm{ZnFe}$ imersa em solução de $\mathrm{Ce}\left(\mathrm{NO}_{3}\right)_{3}$ e liga $\mathrm{ZnFe}$ imersa em solução de $\mathrm{La}\left(\mathrm{NO}_{3}\right)_{3}$ secadas a $100^{\circ} \mathrm{C}$ por 40 minutos, objetivando comprovar a presença de $\mathrm{CeO}_{2}$, $\mathrm{Ce}_{2} \mathrm{O}_{3}$ e $\mathrm{La}_{2} \mathrm{O}_{3}$ sobre a superfície da liga $\mathrm{ZnFe}$.

A seguir serão introduzidos os resultados relacionados aos ensaios de resistência à corrosão por EIE para cada um dos filmes de inibidores aplicado sobre a liga $\mathrm{ZnFe}$ em meio de $\mathrm{NaCl}$ 0,05M e em seguida, uma comparação será feita entre eles com as imagens obtidas por MEV e análises de EDS, antes e depois das 72 horas de ensaio de corrosão em $\mathrm{NaCl} 0,05 \mathrm{M}$. Na sequência virão as análises dos ensaios de polarização relacionadas com os resultados das análises de EIE.

A Tabela 2 apresenta as denominações das amostras para os respectivos filmes e a camada de liga $\mathrm{ZnFe}$. Os resultados de EIE para as amostras serão apresentados de forma temporal com início em $0 \mathrm{~h}$ indo até 72 horas de imersão e sempre em comparação com o primeiro momento do ensaio de corrosão para a liga $\mathrm{ZnFe}$.

Tabela 2: Denominação das amostras.

\begin{tabular}{l|r|r}
\hline AMOSTRAS & \multicolumn{1}{|c|}{$\begin{array}{c}\text { FILMES OBTIDOS } \\
\text { A PARTIR DE: }\end{array}$} & \multirow{2}{*}{ SUBSTRATO } \\
\hline $\mathrm{ZnFe}+\mathrm{Ce}$ & $\mathrm{Ce}_{2} \mathrm{O}_{3} \mathrm{e} \mathrm{CeO}_{2}$ & \multirow{2}{*}{$\mathrm{ZnFe}$} \\
\hline $\mathrm{ZnFe}+\mathrm{La}$ & $\mathrm{La}_{2} \mathrm{O}_{3}$ & \\
\hline
\end{tabular}




\subsection{Caracterização por XPS}

Os primeiros resultados a serem apresentados são os de XPS. A partir desses resultados é que foi possível mostrar a presença de $\mathrm{CeO} 2, \mathrm{Ce} 2 \mathrm{O} 3$ e $\mathrm{La} 2 \mathrm{O} 3$ sobre a superfície da liga $\mathrm{ZnFe}$.

Como os filmes de óxidos formados sobre a superfície da liga $\mathrm{ZnFe}$ são bastante finos a análise por XPS foi fundamental nesse estudo, pois essa técnica é capaz de analisar as primeiras camadas atômicas (20$50 \AA$ ), tanto de composição elementar como de ambiente químico.

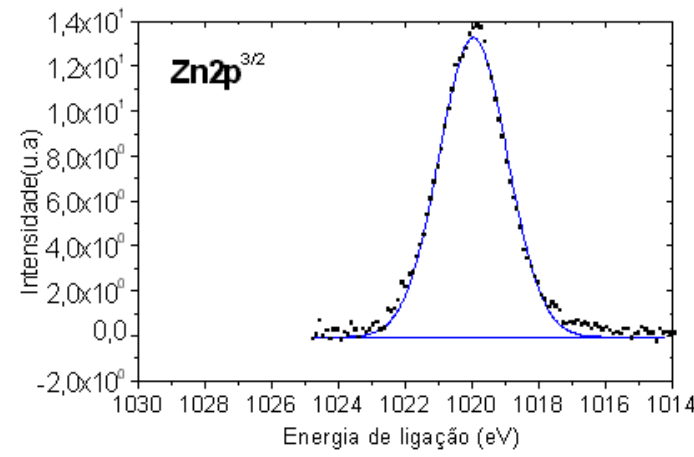

(a)

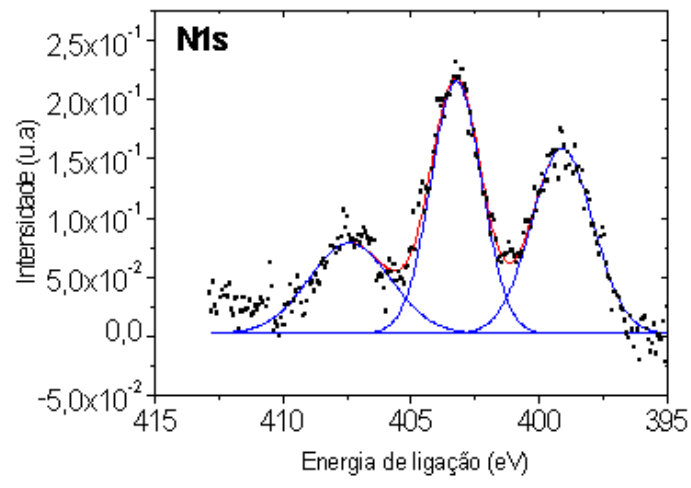

(c)

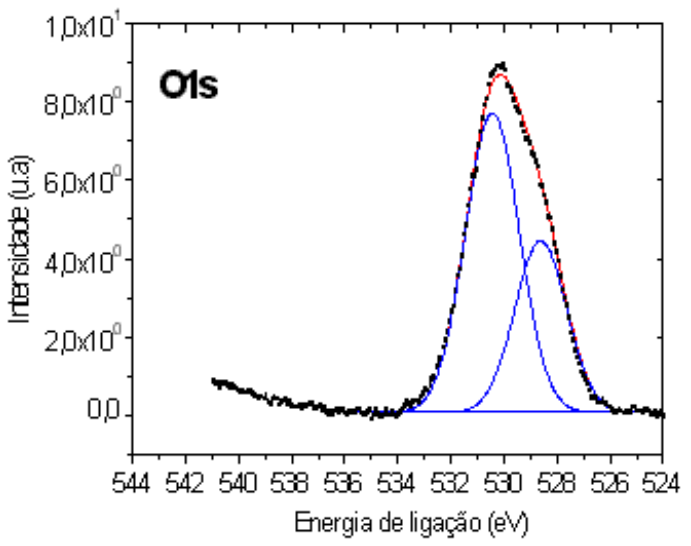

(b)

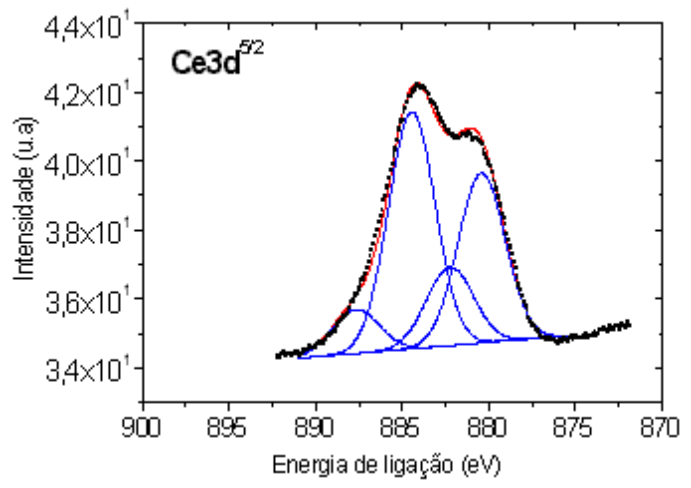

(d)

Figura 1: Espectros de XPS para $\mathrm{Zn} 2 \mathrm{p}^{3 / 2}, \mathrm{O} 1 \mathrm{~s}, \mathrm{~N} 1 \mathrm{~s}$ e $\mathrm{Ce} 3 \mathrm{~d}^{5 / 2}$ obtidos sobre a superfície da liga $\mathrm{ZnFe}$ tratada com $\mathrm{Ce}\left(\mathrm{NO}_{3}\right)_{3}$ e curada a $100^{\circ} \mathrm{C}$ por 40 minutos.

Os espectros de $\mathrm{Zn2p}$, O1s, N1s e Ce3d obtidos por XPS sobre a superfície da liga ZnFe tratada com soluções contendo $\mathrm{Ce}\left(\mathrm{NO}_{3}\right)_{3} \mathrm{e} \mathrm{La}\left(\mathrm{NO}_{3}\right)_{3}$ e curadas a $100^{\circ} \mathrm{C}$ por 40 minutos são apresentados nas Figuras $1 \mathrm{e}$ 2.

As Figuras 1a e 2a mostram os espectros de $\mathrm{Zn} 2 \mathrm{p}^{3 / 2}$ relativos ao estado químico do $\mathrm{Zn}$ e como pode ser observado em $1020 \mathrm{eV}$ o zinco se apresenta na forma $\mathrm{Zn} 0$. As Figuras $1 \mathrm{~b}$ e $2 \mathrm{~b}$ apresentam os espectros de $\mathrm{O} 1 \mathrm{~s}$ e em $530 \mathrm{eV}$ o oxigênio mostra-se na forma de $\mathrm{O}^{-2}$. 


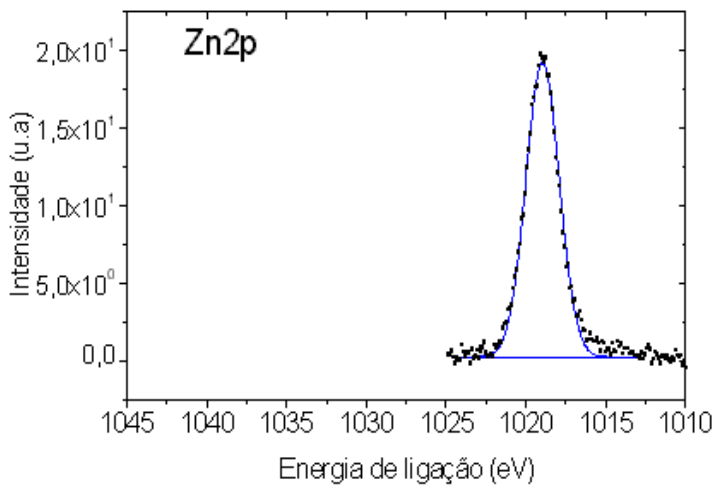

(a)

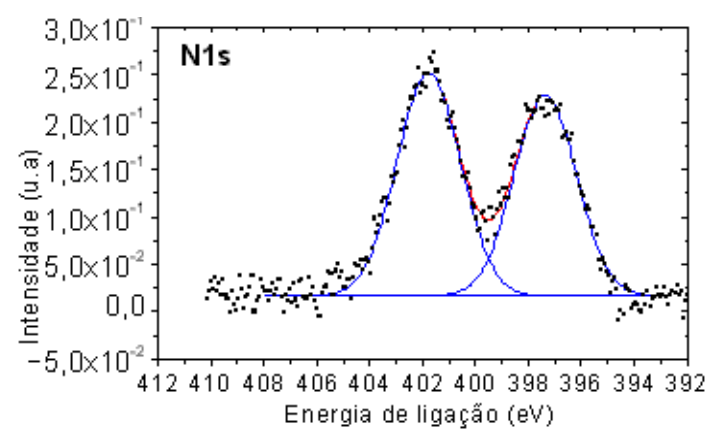

(c)

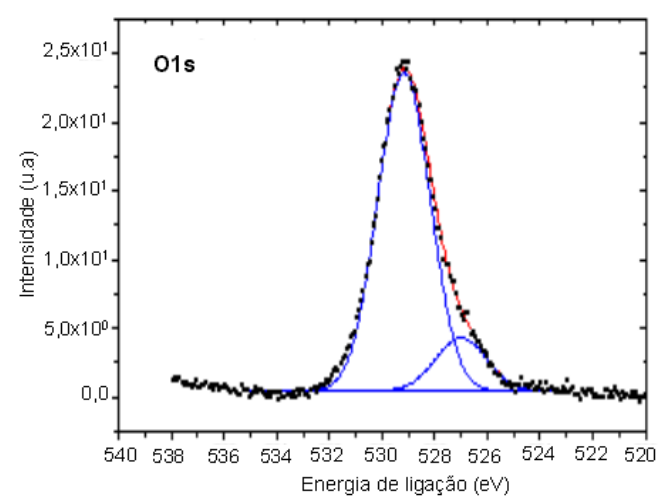

(b)

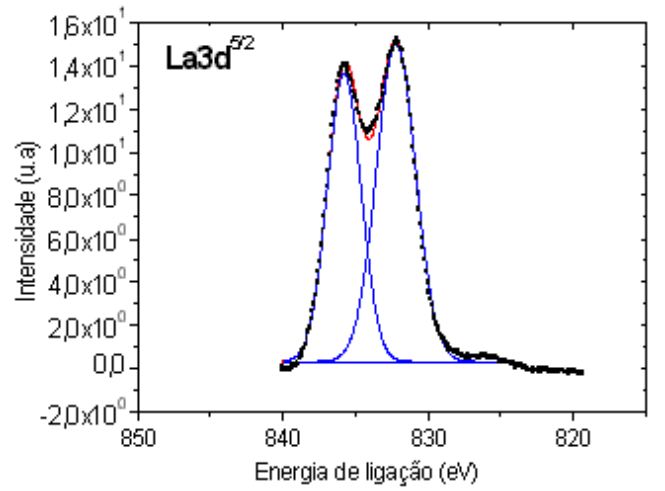

(d)

Figura 2: Espectros de XPS para $\mathrm{Zn} 2 \mathrm{p}^{3 / 2}, \mathrm{O} 1 \mathrm{~s}, \mathrm{~N} 1 \mathrm{~s}$ e $\mathrm{Ce} 3 \mathrm{~d}^{5 / 2}$ obtidos sobre a superfície da liga $\mathrm{ZnFe}$ tratada com $\mathrm{La}\left(\mathrm{NO}_{3}\right)_{3}$ e curada a $100^{\circ} \mathrm{C}$ por 40 minutos.

A Figura 1c apresenta o espectro de N1s, o nitrogênio em $403 \mathrm{eV}$ mostra-se na forma de $\mathrm{NO}^{2-}$ e na Figura $2 \mathrm{c}$ como $\mathrm{NO}^{3-}$ e $\mathrm{NO}^{2-}$ em $407 \mathrm{e} 403 \mathrm{eV}$ respectivamente. Nota-se, no entanto, que somente traços desses ânions foram detectados.

A Figura $1 \mathrm{~d}$ apresenta o espectro de $\mathrm{Ced}^{5 / 2} \mathrm{em} 880$ e $885 \mathrm{eV}$ o cério mostra-se na forma de $\mathrm{Ce}_{2} \mathrm{O}_{3}$ e em $882 \mathrm{eV}$ na forma de $\mathrm{CeO}_{2}$ segundo TROMAT et. al [16].

A Figura $2 \mathrm{~d}$ apresenta o espectro de $\mathrm{La}^{3 / 2}$ que em $832 \mathrm{eV}$ esta na forma de $\mathrm{La}_{2} \mathrm{O}_{3}$. HOUNG, et. al [17].

\subsection{Caracterização dos Filmes por EIE, MEV e EDS}

As Figuras 3 e 4 apresentam os resultados de EIE obtidos para as amostras $\mathrm{ZnFe}+\mathrm{Ce}$ e $\mathrm{ZnFe}+\mathrm{La}$ em diferentes tempos de ensaio de imersão em solução de $\mathrm{NaCl}$ 0,05M respectivamente. Como podem ser observadas nos diagramas, as amostras contendo os sais de terras raras (TR) apresentam valores de módulo de impedância maiores que os encontrado para a liga $\mathrm{ZnFe}$ no primeiro momento de reação $(\mathrm{t}=0)$, Figuras $3 \mathrm{a}$ e 4a. Esse comportamento pode ser resultado de reações que estão ocorrendo nas superfícies, como formação de óxidos/hidróxidos, (por causa dos átomos de $\mathrm{Ce}$ e La ou mesmo dos elementos constituintes da liga eletrodepositada sobre o aço carbono, ou seja, $\mathrm{Zn}$ e o Fe).

Esse resultado no módulo de $\mathrm{Z}$ reflete no ângulo de fase, pois a partir de T4 surgem duas constantes de tempo (Figura $4 \mathrm{~b}$ ). Nos dois primeiros tempos de imersão há um ligeiro deslocamento do pico de ângulo 
de fase no eixo da frequência em relação à amostra de liga $\mathrm{ZnFe}$; além disso, há um pequeno aumento no valor do ângulo de fase. Esse resultado pode estar relacionado a alterações do filme contendo óxidos de Ce3+/Ce4+ na superfície. Com o passar do tempo de reação essa camada contendo o cério parece sofrer alterações/reações as quais são indicadas pela presença das duas constantes de tempo mencionadas anteriormente. Ainda, com o passar do tempo de reação novamente vai ocorrendo um deslocamento na freqüência onde aparece o maior pico de ângulo de fase, que vai se deslocando para frequências próximas a camada de liga $\mathrm{ZnFe}$, ou seja, quanto mais longo o tempo de reação o substrato tende a ficar exposto.

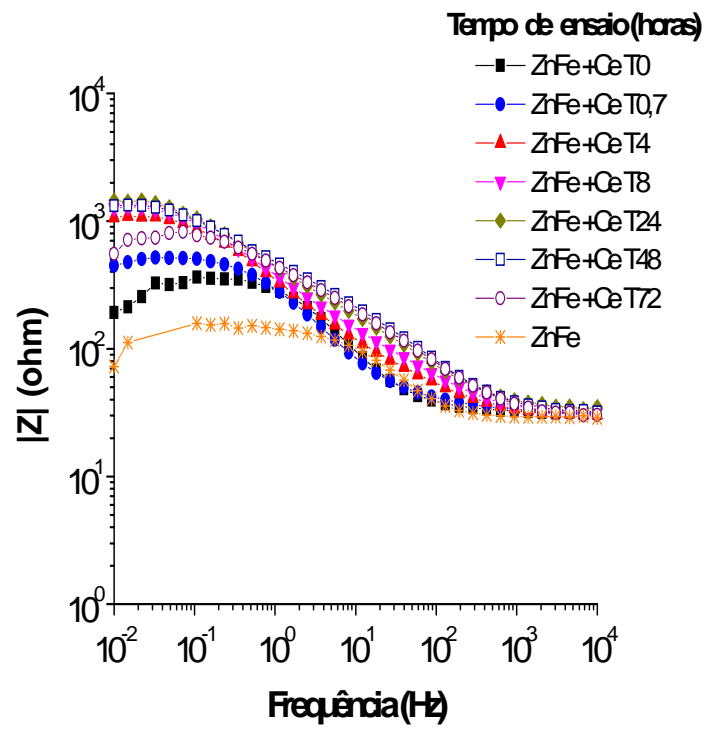

(a)

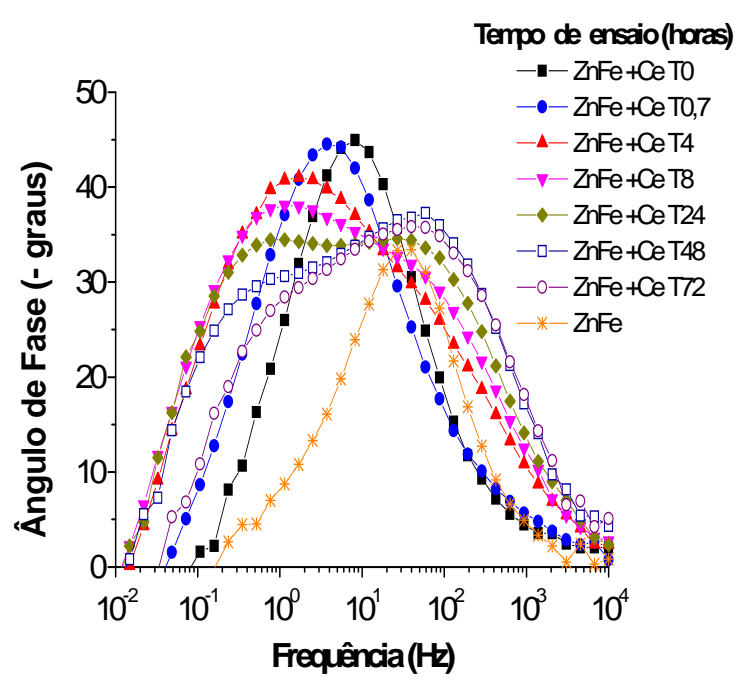

(b)

Figura 3: Diagrama de Bode para a liga de $\mathrm{ZnFe}+\mathrm{Ce}$ em vários tempos de ensaio de corrosão e para a liga $\mathrm{ZnFe}$ no primeiro tempo de ensaio $(\mathrm{t}=0)$.

Os mecanismos de ação inibidora com o uso de TR vêm sendo comprovados com diversas referências bibliográficas relacionadas à proteção à corrosão do zinco [6,9] e ligas de alumínio [2]. As propriedades inibidoras de corrosão do cério podem ser explicadas devido à formação de filmes de óxidos/ hidróxidos de cério misturados a pequenas quantidades de compostos de zinco inibindo as reações catódicas, segundo estudos de ARAMAKI [9], ALDYKEWICZ, et al. [2].

A Figura 4 apresenta o comportamento do filme contendo os átomos de La sobre a liga $\mathrm{ZnFe}$ com o passar do tempo e indica um comportamento semelhante ao do Ce porém com valores de $|Z|$ e ângulos de fase menos expressivos, sendo valor de IZI em baixa freqüência para $\mathrm{ZnFe}+\mathrm{La}$ de $400 \mathrm{ohm}$ e em 72 hora 500 ohm . Essa menor expressividade pode estar relacionada a reações mais rápidas e/ou produtos de reações menos protetores. No final da imersão, vê-se que os valores de ângulos de fase estão em média $50 \%$ mais baixos, indicativos de reações.

Íons de La podem ser usados como inibidores de soluções aquosas para proteção do Alumínio, Magnésio [18, 19]. A proteção a corrosão da liga $\mathrm{ZnFe}$ na presença de átomos de La pode ser atribuída a formação de um filme composto de $\mathrm{La}_{2} \mathrm{O}_{3}, \mathrm{La}(\mathrm{OH})_{3}$ e pequenas quantidades de $\mathrm{Zn}(\mathrm{OH})_{2}$ e $\mathrm{ZnO}$ e os resultados de impedância indicam esse comportamento. 


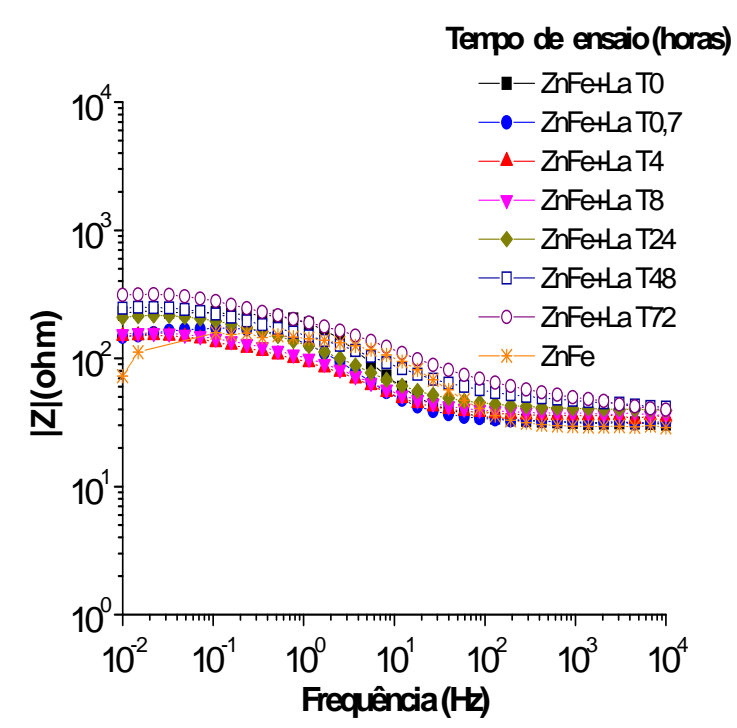

(a)

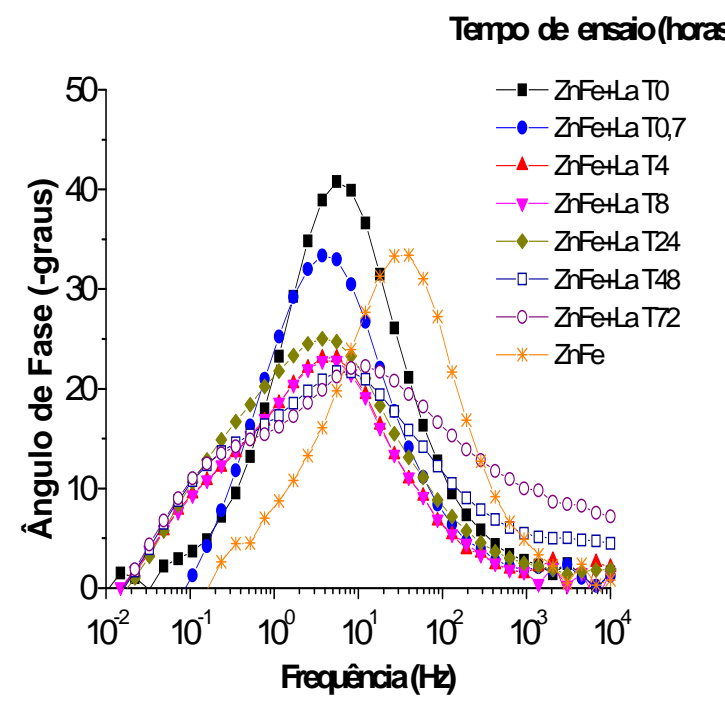

(b)

Figura 4: Diagrama de Bode para a liga de $\mathrm{ZnFe}+\mathrm{La}$ em vários tempos de ensaio de corrosão e para a liga $\mathrm{ZnFe}$ no primeiro tempo de ensaio $(\mathrm{t}=0)$.

As Figuras 5 e 6 apresentam as micrografias das amostras $\mathrm{ZnFe}+\mathrm{Ce}$ e $\mathrm{ZnFe}+\mathrm{La}$ antes e depois dos ensaios de corrosão em solução de $\mathrm{NaCl}$ 0,05M. Como pode ser visto, as superfícies das amostras antes dos ensaios apresentam-se sem nenhum defeito aparente. Após 72 horas de reação a amostra $\mathrm{ZnFe}+\mathrm{Ce}$ apresenta vários, porém pequenos pontos negros espalhados de forma homogênea, como podem ser observados na Figura $5 b$.

A técnica de EDS não é ideal para a detecção dos elementos leves, e as microanálises realizadas nas amostras foram úteis, no sentido de se conhecer os elementos químicos presentes. Através das análises pontuais pode-se assumir oxidação nos pontos 1, 2 e 3 que na Tabela 3 pode estar indicando a formação de $\mathrm{Ce}_{2} \mathrm{O}_{3}$ e $\mathrm{Ce}(\mathrm{OH})_{3}$ sobre as regiões catódicas, formando assim um filme de óxidos que fosse capaz de impedir a continuidade da reação de redução de oxigênio.

Tabela 3: Análise de EDS Concentração Atômica (\%) da amostra de ZnFe+ Ce. após 72 horas d e ensaio de corrosão.

\begin{tabular}{c|c|c|c|c}
\hline ZnFe+Ce & O-K & Fe-K & Zn-K & Ce-L \\
\hline ZnFe+Ce_pt1 & 33,12 & 36,76 & 29,95 & 0,17 \\
\hline ZnFe+Ce_pt2 & 30,75 & 40,20 & 28,86 & 0,18 \\
\hline ZnFe+Ce_pt3 & 43,77 & 28,56 & 27,33 & 0,34 \\
\hline ZnFe+Ce_pt4 & 14,26 & 12,44 & 73,00 & 0,30 \\
\hline
\end{tabular}




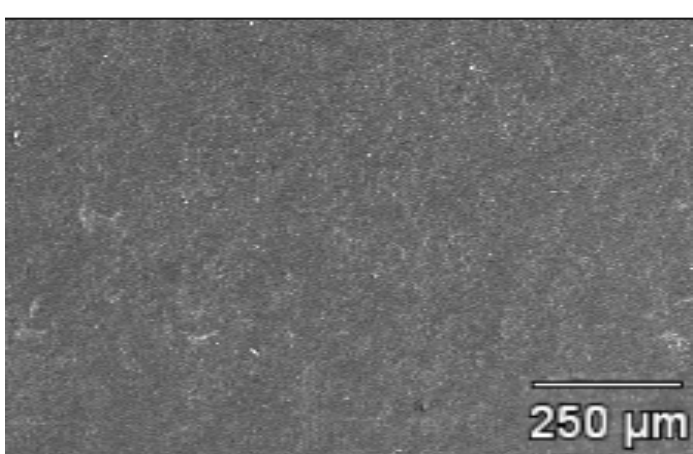

(a)

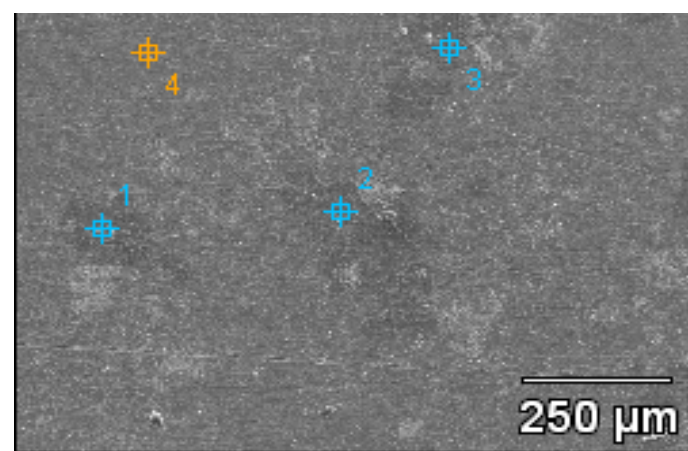

(b)

Figura 5: Imagens obtidas por MEV da amostra de ZnFe + Ce (a) sem corrosão; (b) 72horas de ensaio de corrosão. Aceleração de voltagem: $5 \mathrm{kV}$ e $25 \mathrm{kVrespectivamente.}$

A Figura 6b apresenta a micrografia da superfície da amostra $\mathrm{ZnFe}+\mathrm{La}$ após 72 horas de ensaio de corrosão em solução de $\mathrm{NaCl}$, podendo-se observar pontos de oxidação no formato de largos círculos e bastante espalhada pela amostra, o que corrobora com as informações fornecidas pelos diagramas de impedância. Na Figura 4(b), nota-se após 48 horas de ensaio, ocorre o surgimento de uma segunda constantes de tempo em baixas frequências, sugerindo a formação de compostos como $\mathrm{La}_{2} \mathrm{O}_{3}, \mathrm{La}(\mathrm{OH})_{3}$ e pequenas quantidades de $\mathrm{Zn}(\mathrm{OH})_{2}$ e $\mathrm{ZnO}$. Através das análises pontuais na Tabela 4, essa oxidação pode estar ocorrendo nos pontos 1 e 2 visto que, ocorre um aumento da quantidade relativa de oxigênio e diminuição da quantidade relativa de $\mathrm{Zn}$ nessas regiões. O ponto 3 apresenta-se praticamente intacto, devido à alta quantidade relativa de $\mathrm{Zn}$ e pequena de $\mathrm{Fe}$.

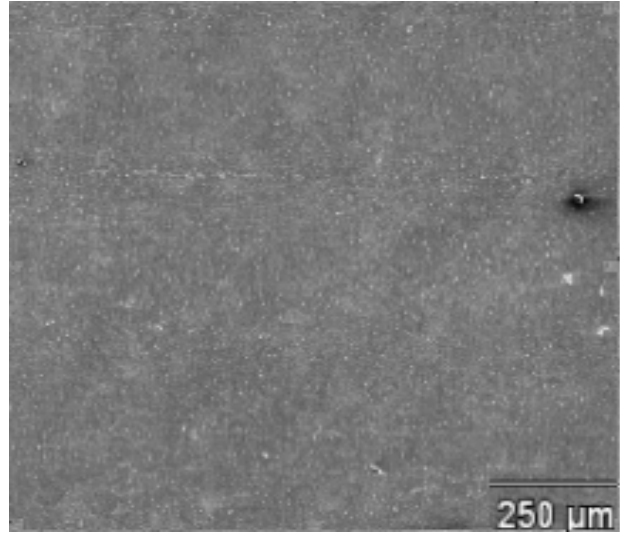

(a)

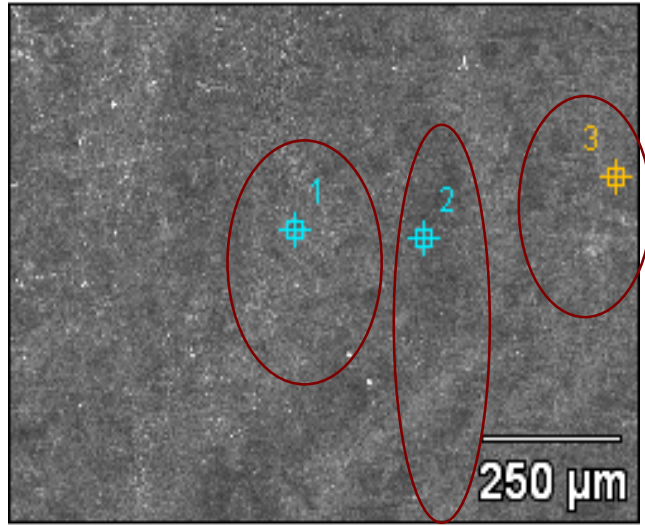

(b)

Figura 6: Imagens obtidas por MEV da amostra de $\mathrm{ZnFe}+\mathrm{La}$ (a) sem corrosão; (b) 72horas de ensaio de corrosão. Aceleração de voltagem: $5 \mathrm{kV}$ e $25 \mathrm{kV}$ respectivamente. 
Tabela 4: Analise de EDS Concentração Atômica (\%) da amostra de $\mathrm{ZnFe}+$ La após 72 horas de ensaio de corrosão.

\begin{tabular}{c|c|c|c|c}
\hline ZnFe+ La & O-K & Fe-K & Zn-K & La -L \\
\hline ZnFe+ La_pt1 & 35,68 & 40,87 & 43,00 & 0,25 \\
\hline ZnFe+ La_pt2 & 32,12 & 50,04 & 17,60 & 0,24 \\
\hline ZnFe+ La_pt3 & 20,99 & 8,70 & 70,20 & 0,11 \\
\hline
\end{tabular}

As Figuras 7 e 8 apresentam as analises por EDS, que acoplado ao MEV, permitiu a realização da microanálise semiquantitativa sobre as amostras buscando dessa forma, conhecer a distribuição dos átomos nos sistemas estudados antes dos ensaios de corrosão.

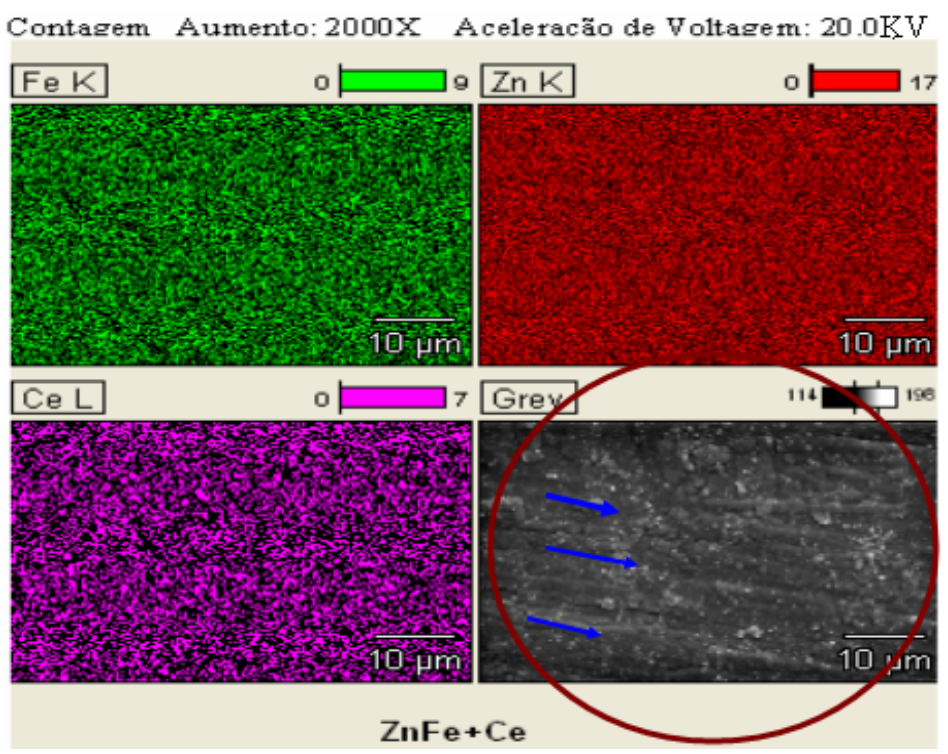

Figura 7: Mapeamento por EDS da superfície da amostra de ZnFe+ Ce antes do ensaio de corrosão.

No mapeamento apresentado na Figura 7, pode-se notar uma distribuição bem homogênea dos elementos por toda a superfície da amostra. A micrografia circulada mostra o relevo da superfície com ausência do mapeamento. Nota-se uma preferência horizontal na deposição dos filmes de óxidos; obtidos pelo processo de imersão da liga $\mathrm{ZnFe}$ na solução contendo $\mathrm{Ce}^{3+}$, e essa morfologia é corroborada pelos estudos realizados por LUMPP [20].

Tabela 5: Análise de EDS Concentração Atômica (\%) da amostra de $\mathrm{ZnFe}+\mathrm{Ce}$, antes do ensaio de corrosão.

\begin{tabular}{c|c|c|c}
\hline ELEMENTOS & QUANTIDADE & CONC \% POR PESO & CONCENTRAÇÃO \% ATÔMICA \\
\hline Fe K & 7464 & 6,84 & 8,00 \\
\hline Zn K & 43291 & 91,28 & 91,13 \\
\hline Ce L & 1617 & 1,87 & 0,87 \\
\hline Total & & 100,00 & 100,00 \\
\hline
\end{tabular}




\section{Contagem Aumento 2000X Voltagem 20KV}

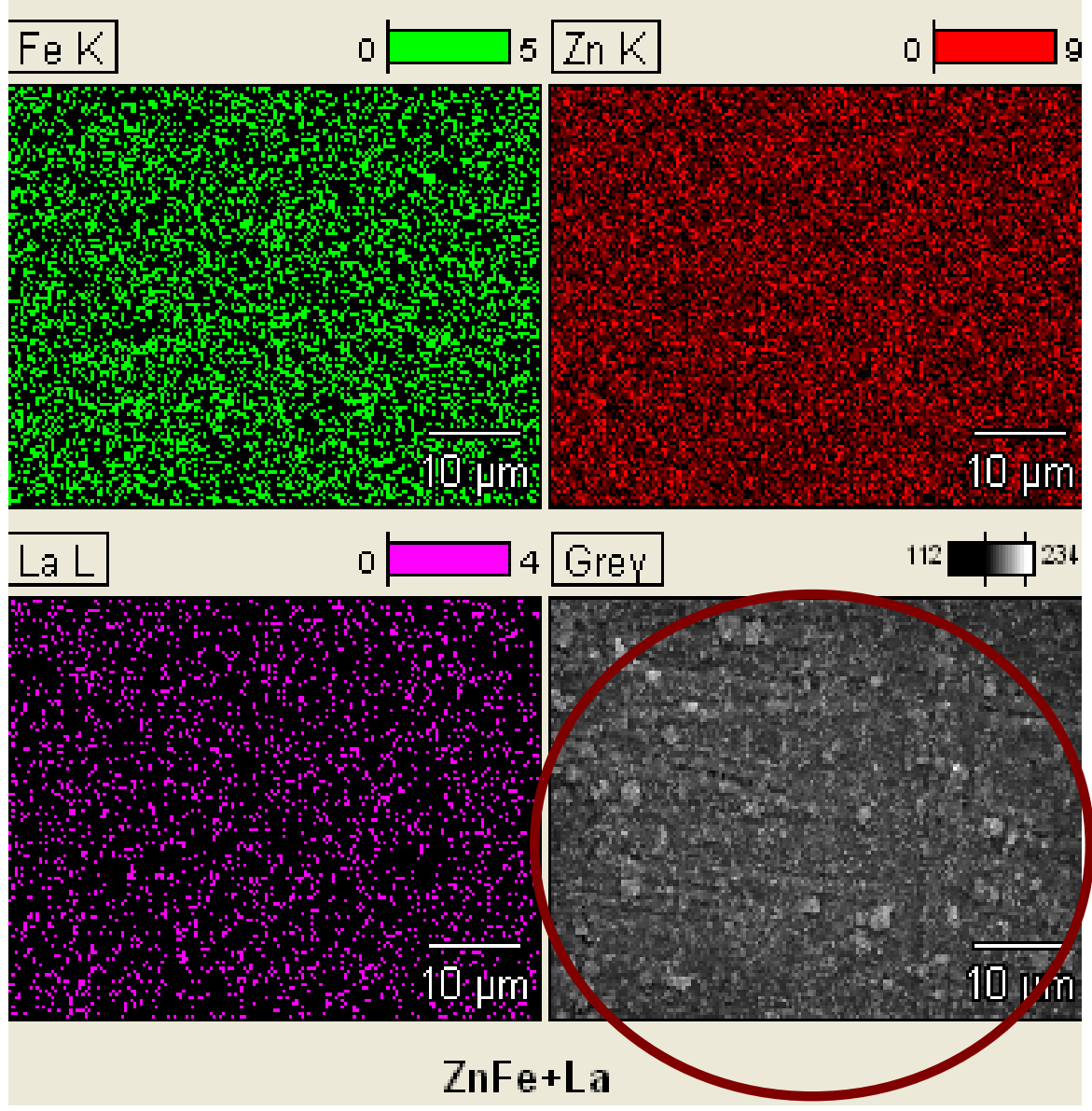

Figura 8: Mapeamento por EDS da superfície da amostra de $\mathrm{ZnFe}+\mathrm{La}$ antes do ensaio de corrosão.

No mapeamento apresentado pela Figura 8 para o sistema $\mathrm{ZnFe}+\mathrm{La}$, pode-se notar uma distribuição bem homogênea dos elementos por toda a superfície da amostra como no caso da amostra $\mathrm{ZnFe}+\mathrm{Ce}$. Uma observação mais cuidadosa revela pequenas áreas não recobertas pelos elementos e através da comparação com a micrografia circulada pode-se notar, que onde existe diferentes grãos formados durante a deposição da liga $\mathrm{ZnFe}$ sobre o aço carbono é justamente a área não recoberta por esses elementos

Na Tabela 6 observam-se às médias dos valores quantificados por EDS. A quantidade relativa de Fe e Zn confirma o depósito realizado da liga sobre o aço carbono e a quantidade relativa de lantânio indica sua existência no sistema.

Tabela 6: Análise de EDS Concentração Atômica (\%) da amostra de $\mathrm{ZnFe}+\mathrm{La}$ antes do ensaio de corrosão.

\begin{tabular}{c|c|c|c}
\hline ELEMENTOS & QUANTIDADE & CONC \% PESO & CONCENTRAÇÃO \%ATOMICA \\
\hline Fe K & 1732 & 7,94 & 9,22 \\
\hline Zn K & 8582 & 90,90 & 90,24 \\
\hline La L & 208 & 1,16 & 0,54 \\
\hline Total & & 100,00 & 100,00 \\
\hline
\end{tabular}

A Figura 9, apresenta as micrografias das amostras de $\mathrm{ZnFe}, \mathrm{ZnFe}+\mathrm{Ce}$ e $\mathrm{ZnFe}+\mathrm{La}$ respectivamente antes dos ensaios de corrosão. A Figura mostra três superfícies com características bastante distintas entre si. 
A simples observação das micrografias fornece informações a respeito das modificações proporcionadas sobre a liga $\mathrm{ZnFe}$ realizadas pela adição dos inibidores. Assim sendo, a adição dos inibidores fornece uma primeira camada capaz de recobrir possíveis imperfeições como poros e trincas que possam por ventura existir na superfície da camada de liga $\mathrm{ZnFe}$.

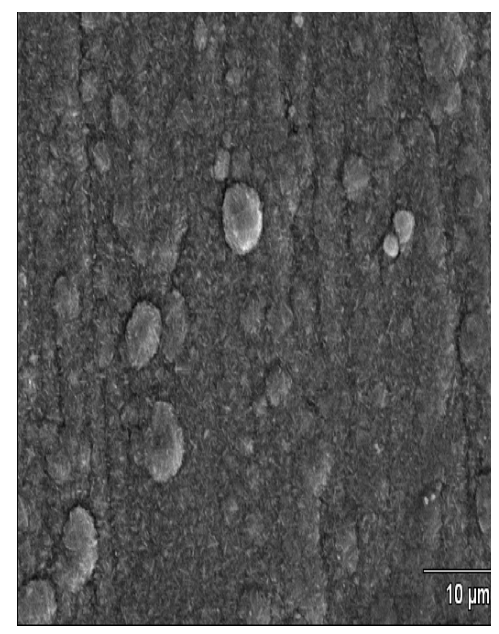

(a)

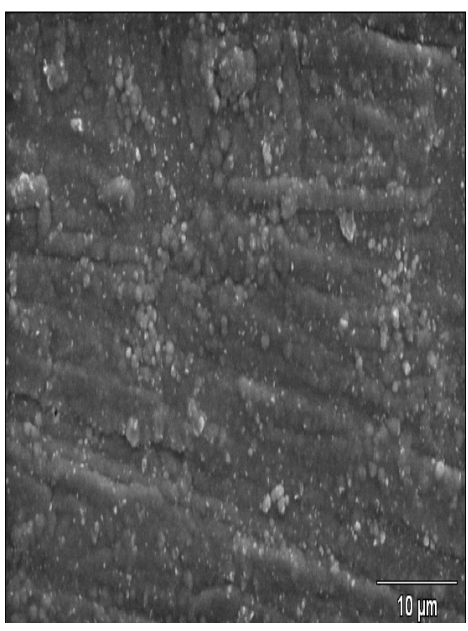

(b)

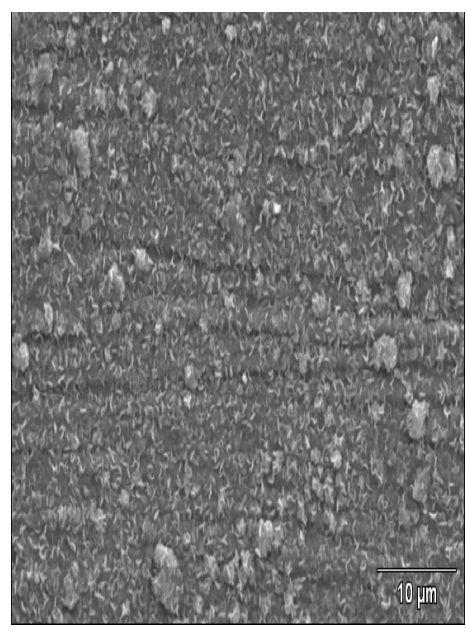

(c)

Figura 9: Imagens obtidas por MEV da superfície das amostras antes dos ensaios de corrosão: (a) liga de $\mathrm{ZnFe}$; (b) $\mathrm{ZnFe}$ + Ce e (c) $\mathrm{ZnFe}+\mathrm{La}$. Aceleração de voltagem: $25 \mathrm{kV}$.

O comportamento eletroquímico da liga $\mathrm{ZnFe}$ e das amostras analisadas anteriormente por EIE, foram avaliados através da técnica de polarização potenciodinâmica.

A Figura 10 apresenta as curvas de polarização anódicas e catódicas para a liga $\mathrm{ZnFe}$ e para as amostras $\mathrm{ZnFe}+\mathrm{Ce}$ e $\mathrm{ZnFe}+\mathrm{La}$ que conforme os resultados apresentados por EIE nos ensaios temporais de imersão em $\mathrm{NaCl}$ 0,05M anteriormente apresentados também indicam características inibidoras de corrosão se comparadas a liga $\mathrm{ZnFe}$ somente.

Os resultados mostraram que os tratamentos realizados provocaram um deslocamento do ramo anódico da curva de polarização de aproximadamente 7 vezes na direção de menores valores de densidade de corrente; entretanto, o efeito resistivo dos filmes na região catódica foi mais marcante.

O ramo catódico da curva de polarização apresentou decréscimo de aproximadamente 10 vezes na densidade de corrente quando a liga $\mathrm{ZnFe}$ foi tratada com as terras raras, podendo-se observar o domínio catódico no processo eletroquímico. A diminuição na densidade de corrente, quando comparada à liga $\mathrm{ZnFe}$, é evidente para as duas amostras. O potencial de corrosão Ecorr apresentou um pequeno deslocamento de $-0,92$. 


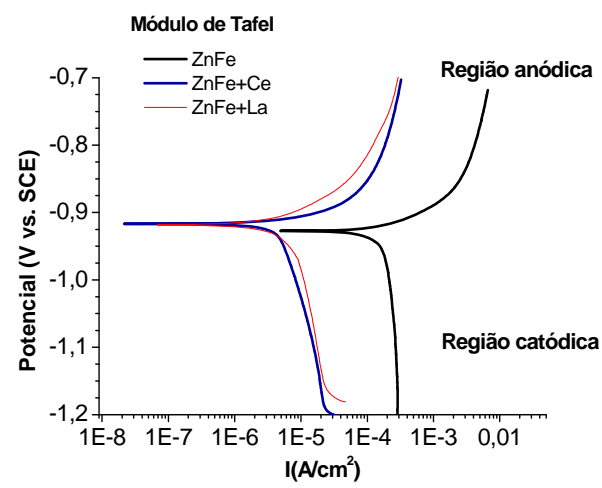

Figura 10: Curvas de polarização da liga $\mathrm{ZnFe}$ e das amostras de $\mathrm{ZnFe}+\mathrm{Ce}$ e $\mathrm{ZnFe}+\mathrm{La}$ ensaiadas em solução de $\mathrm{NaCl}$ 0,05M. (tempo de ensaio $=20$ minutos).

O potencial de corrosão apresentou um pequeno deslocamento de aproximadamente $-10^{-2}$ na direção de maiores potenciais. A densidade de corrente de corrosão variou em aproximadamente 1 ordem de grandeza na direção dos menores valores para aliga revestida com as camadas passivas.

\section{CONCLUSÕES}

$\mathrm{O}$ tratamento usando os óxidos de $\mathrm{Ce}$ ou La aumentou a proteção à corrosão comparativamente a liga $\mathrm{ZnFe}$ sem tratamento;

Os ensaios de EIE foram muito eficientes na caracterização contra corrosão dos sistemas estudados, sendo capaz de evidenciar o melhor filme, ou seja, o que continha os óxidos de cério.

As curvas de polarização de uma maneira geral, mostraram que os tratamentos realizados provocaram diminuição das densidades de corrente nas curvas anódicas; entretanto, a diminuição das densidades de correntes nas curvas catódicas foi mais acentuada, comprovando o efeito resistivo dos filmes.

\section{AGRADECIMENTOS}

Os autores agradecem a CNPq pelo apoio financeiro, e as empresas Tecnorevest pela doação do banho de $\mathrm{ZnFe}$.

\section{BIBLIOGRAFIA}

[1] OJEC- Official Journal of the Europe Communities - End-Of-Life Vehicles - Directive 2000/53/ec of the European Parliament and of the Concil, 18- setembro 2000.

[2] ALDYKIEWICZ, A.J., DAVENPORT, A.J., ISAACS, H.S., “ Studies of the formation of cerium-rich protective films using X-ray absorption near-edge spectrocopy and rotating disk electrode methods", Journal of Electrochemical Society, v. 143, n. 1, pp. 147-154, 1996.

[3] PALOMINO, L.M., AOKI, I. V., DEMELO, H. G., "Microstructural and electrochemical characterization of Ce conversion layers formed on Al alloy 2024-T3 covered with Cu-rich smut", Electrochimica Acta, v. 51, pp. 5943-5953, 2006.

[4] ANDREATTA, F., DRUART, M. E., LANZURTTI, A., et al., "Localized corrosion inhibition by cerium species on clad AA2024 aluminium alloy investigated by means of electrochemical micro-cell”, Corrosion Science, v.65, pp. 376-386, December 2012.

[5] TAKEUCHI, S.M,T., AZAMBUJA, D.S., COSTA I., "Cerium conversion layer for improving the corrosion resistance of phosphated NdFeB magnets", Surface and Coatings Technology., v. 201, n. 6, pp. 3670-3675, December 2006. 
[6] SANTOS M.C.G., Avaliação do desempenho de filmes contendo silanos e sais de terras raras para proteção contra corrosão de camadas ZnFe eletrodepositadas, D.Sc., pp.130, Faculdade de Engenharia Mecânica, Universidade Estadual de Campinas, Campinas -SP, 2005.

[7] ARAMAKI, K., "The inhibition effects of cation inhibitors on corrosion of zinc in aerated $0.5 \mathrm{M} \mathrm{NaCl}$ ", Corrosion Science, v. 43, pp. 591-604, July 2001.

[8] ARAMAKI, K., "Preparation of self-healing protective films on a zinc electrode treated in a cerium (III) nitrate solution and modified with sodium phosphate and cerium (III) nitrate", Corrosion Science, v. 46, pp. 1565-1579, September 2003.

[9] ARAMAKI, K., "Treatment of zinc surface with cerium (III) nitrate to prevent zinc corrosion in aerated 0.5M NaCl”, Corrosion Science, v. 43, pp. 2201-2215, November 2001.

[10] MONTEMOR M.F., SIMÔES A.M.P., FERREIRA M.G.S., "Composition and behaviour of cerium films on galvanised steel”, Progress in Organic Coatings, v. 43, n. 4, pp. 274-281, December 2001.

[11] FERREIRA, M.G.S., DUARTE, R.G., MONTEMOR, M.F., et al., "Silanes and rare earth salts as chromate replacers for pre-treatments on galvanized steel”, Electrochimica acta, v.49, pp.2927-2935, 2004.

[12] PENG, T., MAN, R., "Rare earth and silane as chromate replacers for corrosion protection on galvanized steel", Journal of rare Earths, v.27, pp.159-163, 2009.

[13] BALASUBRAMANIAM, M., MELENDRES, C.A., MANSOUR, A.N., "An X-ray absorption study of the local structure of cerium in electrochemically deposited thin films", Thin Solid Films, v. 347, pp. 178-183, 1999.

[14] TAMBORIM, S.M., MAISONAVE, A.P.Z., AZAMBUJA, D.S., et al., "An electrochemical and superficial assessment of the corrosion behavior of AA 2024-T3 treated with etacryloxypropylmethoxysilane and cerium nitrate", Surface and Coatings Technology, v.202, n.24, pp. 5991-6001, August 2008.

[15] KAUTEC W., SHARE M., PAATSH W., "Transition metal effects in the corrosion protection of elestroplated zinc alloy coatings", Electrocchimica Acta, v.39, pp.1151-1157,1994.

[16] TROMAT, N., GAUTIER-SOYER, M., BORDIER, G., "Formation of the Ce/Y2O3 interface: an in XPS study", Surface Science, v.345, pp. 290-302, 1996.

[17] HOWNG, W.Y., THORN, R.J., "Investigation of the electronic structure of La1- X (M+2)XCrO3, Cr2O3 and La2O3 by X-Ray photoelectron-spectroscopy", Journal Physics and Chemistry of Solids, v.41, n.1, pp. 75-81, 1980.

[18] ABALLE A., BENTHECOURT M., BOTANA F.J., et al.," $\mathrm{CeCl} 3$ and $\mathrm{LaCl} 3$ binary solutions as environment-friendly corrosion inhibitors of AA5083 Al-Mg alloy in $\mathrm{NaCl}$ solutions", Journal of Alloys and Compounds, v. 323-324, pp. 855-858, 2001.

[19] RUDD, A.L., BRESLID, C.B., MANSFELD, F., "The corrosion protection afforded by rare earth conversion coatings applied to magnesium", Corrosion Science, v.42, n.1-2, pp.275-288, 2000.

[20] LUMPP, S.N., Obtenção de filmes passivantes de molibidato de amônio e nitrato de cério para ligas de zinco eletrodepositadas, D.Sc., pp.130, Faculdade de Engenharia Mecânica, Universidade Estadual de Campinas, Campinas-SP, 2005. 